\title{
Method for processing XCP data with improved accuracy
}

\author{
Xinyue Zhang ${ }^{1}$, Qisheng Zhang ${ }^{1}$, Xiao Zhao ${ }^{1}$, Qimao Zhang ${ }^{2}$, Shenghui Liu ${ }^{1}$, Shuhan Li $^{1}$, and Zhenzhong Yuan ${ }^{1}$ \\ ${ }^{1}$ School of Geophysics and Information Technology, China University of Geosciences (Beijing), Beijing, 100083, China \\ ${ }^{2}$ Institute of Electronics, Chinese Academy of Sciences, Beijing, 100190, China
}

Correspondence to: Qisheng Zhang (zqs@ @ugb.edu.cn)

Received: 17 December 2016 - Discussion started: 30 January 2017

Revised: 29 March 2017 - Accepted: 24 April 2017 - Published: 16 May 2017

\begin{abstract}
An expendable current profiler (XCP) is a device used for monitoring ocean currents. In this study, we focus on the technology available for processing XCP data and propose a more accurate method for calculating the current velocity from the nanovolt-scale current-induced electric field measured using an XCP. In order to confirm the accuracy of the proposed data processing method, a sea test was performed in the South China Sea region, wherein, for the first time in China, ocean current and electric-field data were collected from the sea surface to a depth of $1000 \mathrm{~m}$ using an XCP. The current-data processing method described herein was used to determine the eastward and northward relative velocity components of the current from the measured data, which were then compared with the current data obtained using an acoustic Doppler current profiler, in order verify the accuracy of the measurements as well as that of the data processing method.
\end{abstract}

\section{Introduction}

Oceans cover approximately 360 million $\mathrm{km}^{2}$ of the earth's surface, thus accounting for $71 \%$ of its surface area. Ocean currents play a significant role in various geological, physical, chemical, and biological processes as well as in the formation of the surrounding climates and weather patterns and the variability seen in them. Therefore, elucidating the laws that govern ocean currents and their patterns is of great importance to the fishing and shipping industries as well as from a military point of view (Crews and Futterman, 1962).

The expendable current profiler (XCP) can gather information related to the current profile quickly (Liu and $\mathrm{He}$, 2010). The XCP is jettisoned from a ship, submarine, aircraft, or similar carrying platform so that it can rapidly mea- sure the current and temperature profiles as it sinks and calculate the corresponding water depth based on the sinking speed (Liu et al., 2007; Chen et al., 2011). The data obtained are transmitted to the carrying platform through a wire or by wireless communication and are processed, yielding the real-time variations in the current and temperature with the depth. XCPs can be used in oceanographic surveying, marine environmental forecasting, scientific research, and military applications and are advanced and highly efficient devices for data collection (Liu and He, 2010; Chen et al., 2010). The XCP is used for monitoring ocean currents, and an XPS was recently developed in China specifically for obtaining current-related data. The XCP works on the following principle: the horizontal motion of the current makes it cut the vertical component of the geomagnetic field, inducing an electric field (Larsen and Sanford, 1985; Zhang et al., 2008; Lin et al., 2003). In a stable geomagnetic field, the magnitude of the induced electric field depends on the velocity of the current (Larsen and Sanford, 1985; Wang et al., 2005; Yan et al., 2011). Therefore, the motion properties of the current can be determined by measuring the generated electric field (Chave et al., 1990; Wu and Chen, 1991; Hisayoshi and Hisashi, 2015). Within a certain temporal and spatial range, the current-induced electric field is a weak direct current (DC) signal (of the order of tens of nanovolts). However, in this case, measuring the nanovolt-scale DC signal becomes a significant challenge. During the measurements corresponding to the sinking process, the above-mentioned XCP probe modulates the nanovolt-scale DC signal into an approximate single-frequency alternating current (AC) signal through mechanical rotation. However, determining the amplitude and phase of such weak single-frequency AC signals is also a technical challenge, making it difficult to process the current data. In order to solve this problem, the XCP uses a voltage- 
to-frequency converter to determine the in-phase component, quadrature component, and baseline data related to the compass coil, along with the induced electric-field signal. In this study, it was assumed that this single-frequency AC signal (coil and induced electric-field signal) is a modulated signal with a carrier frequency, and a method for processing the current data to calculate the amplitude and phase of the modulated signal was used in order to determine the eastward and northward relative velocity components of the current.

In this study, we propose a method for processing XCP current data in order to improve its accuracy. To efficiently calculate the current parameters based on XCP data, two steps are essential. The first is to calculate the electric field that is generated by a given current and use the results of this calculation to determine the speed and direction of the current corresponding to the measured electric field. The second is to determine the effect of placing the probe in seawater on the electric field in order to ensure the accuracy of the current measurements. In addition, owing to the differences in the microchips, capacitors, and resistors used in different $\mathrm{XCP}$ probes, the magnitude of the simulated signal may deviate from the theoretical value; this will directly affect the results of the subsequent data processing. Thus, in order to ensure the accuracy of the XCP data processing, the XCP probe used in this study was calibrated. Finally, the accuracy of the method used for processing the current data was confirmed through a sea test.

\section{Processing of ocean current data obtained using XCP}

\subsection{Principle underlying XCP ocean current data processing}

In a rectangular coordinate system consisting of the $x$ axis (east), $y$ axis (north), and $z$ axis (vertical), an ocean current with velocity $V$ flows horizontally in any direction, and the measurement direction angle between the induced voltage and the $y$ axis is $\theta$. The electromotive force, $\Delta \Phi_{1}$, induced by the ocean current, as measured between two points on horizontally placed electrodes and separated by distance $L$, is given by the following equation (Liu and He, 2010; Larsen and Sanford, 1985):

$$
\begin{aligned}
& \Delta \Phi_{1}=F_{z} \times(\boldsymbol{V}-\overline{\boldsymbol{V}}) \cdot \boldsymbol{L} \\
& =\left(V_{\mathrm{E}}-\overline{\bar{V}}_{\mathrm{E}}\right) F_{z} L \cos \theta-\left(V_{\mathrm{N}}-\bar{V}_{\mathrm{N}}\right) F_{z} L \sin \theta,
\end{aligned}
$$

where $V_{\mathrm{E}}, \bar{V}_{\mathrm{E}}, V_{\mathrm{N}}$, and $\bar{V}_{\mathrm{N}}$ represent the eastward component of the current velocity, average velocity of the eastward component, northward component of the velocity, and average velocity of the northward component, respectively, and $F_{z}$ is the vertical component of the geomagnetic field. Equation (1) describes the electric field induced by an ocean current. From the equation, it can be seen that the measured voltage is proportional not only to the distance $L$ measured by the probe but also to $(\boldsymbol{V}-\overline{\boldsymbol{V}})$, the relative velocity of the ocean cur- rent. Therefore, $(\boldsymbol{V}-\overline{\boldsymbol{V}})$ can be obtained by measuring $\Delta \Phi_{1}$. However, because it is difficult to determine $\bar{V}$ in practice, the measured data for the electromagnetic fields induced by ocean currents are used to calculate the relative velocity of the currents.

Based on the model proposed by Larsen and Sanford (1985), Eq. (1) can be improved by adding a compensation factor for the XCP probe, as shown in Eq. (2), where $K$ is indicative of the impact of the XCP probe on the distribution of the current-induced electric field around the probe when it is placed in seawater. Numerical simulations, forward modelling, and physical simulations have shown that, when the XCP probe is placed in the test area, there is an approximately 2-fold increase in the strength of the currentinduced electric field as measured by the electric-field sensor of the XCP. Thus, the value of $K$ is normally taken to be 1 (Zhang et al., 2016).

The electric field, $\psi_{2}$, generated by the two electrodes cutting through the geomagnetic field during the descent of the $\mathrm{XCP}$ probe is expressed by Eq. (3), where $F_{\mathrm{H}}$ is the horizontal geomagnetic field, $W$ is the descent velocity of the $\mathrm{XCP}$ probe, $L$ is the interelectrode spacing, and $\theta$ is the angle between the measurement electrodes and the $y$ axis (magnetic north). Therefore, the total voltage, $\Delta U_{\mathrm{e}}$, acting on the electric-field sensor of the XCP is given by Eq. (4).

$$
\begin{aligned}
& \Delta \Phi_{1}=(1+K)\left[\left(V_{\mathrm{E}}-\bar{V}_{\mathrm{E}}\right) F_{z} L \cos \theta\right. \\
& \left.-\left(V_{\mathrm{N}}-\bar{V}_{\mathrm{N}}\right) F_{z} L \sin \theta\right] \\
& \psi_{2}=F_{\mathrm{H}} L W \sin \theta \\
& \Delta U_{\mathrm{e}}=\Delta \Phi_{1}+\psi_{2} \\
& =F_{z} L\left(V_{\mathrm{E}}-\bar{V}_{\mathrm{E}}\right)(1+K) \cos \theta \\
& -\left[F_{z}\left(V_{\mathrm{N}}-\bar{V}_{\mathrm{N}}\right)(1+K)-F_{\mathrm{H}} W\right] L \sin \theta
\end{aligned}
$$

$\Delta U_{\mathrm{e}}$ and $\psi_{2}$, namely, the voltage signal measured by the electrodes and the coil signal, are converted into pulse signals by a voltage-frequency converter; the magnitude of the voltage is represented by the magnitude of the frequency of the signal (Zhang et al., 2011). During the measurement process, the modulated signals are demodulated by the probe using electrical circuits, based on the compass coil signals, and the demodulated signals are transmitted to the surface XCP float (Gandolfi et al., 1972). They are then relayed to and stored in the wireless ocean current data receiver in the deck unit.

The modulated signals can be modelled as follows:

$F(t)=A \cos (\omega t+\varphi)+C+D t+E t^{2}+\delta$,

where $\omega$ is the angular frequency of the probe spin; $\varphi$ is its phase position; $C, D$, and $E$ are the delay coefficients of the circuits; and $\delta$ is the measurement noise.

In fact, the primary data recorded by the measurement instruments include the period counts of the useful signals and the coil signals, and the two types of signals are demodulated 


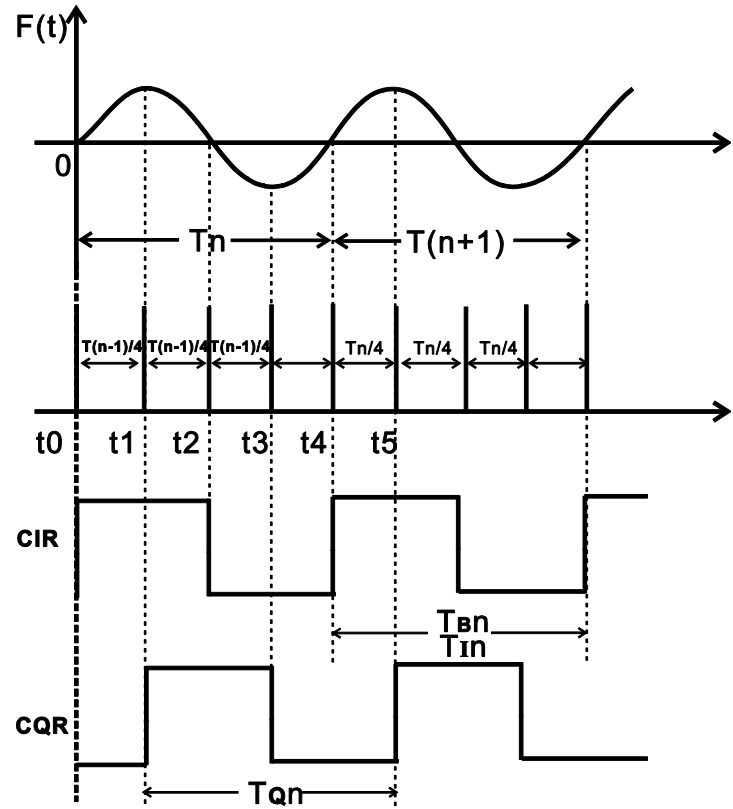

Figure 1. Measurement of period of voltage-frequency converter of $F(t)$.

into their in-phase component $I_{n}$, quadrature component $Q_{n}$, and baseline component $B_{n}$. The measurement of the period of the voltage-frequency converter of $F(t)$ is shown in Fig. 1, where CIR represents the control signals of the inphase component counter and CQR represents the control signals of the quadrature component counter. According to Fig. 1, the relationship between $I_{n}, Q_{n}$, and $B_{n}$ of the modulated signals is as follows:

$$
\begin{array}{r}
I_{n}=\int_{t 0}^{t 2} F(t) \mathrm{d} t-\int_{t 2}^{t 4} F(t) \mathrm{d} t, \\
Q_{n}=\int_{t 1}^{t 3} F(t) \mathrm{d} t-\int_{t 3}^{t 5} F(t) \mathrm{d} t, \\
B_{n}=\int_{t 0}^{t 4} F(t) \mathrm{d} t,
\end{array}
$$

and $t 0=0, t 1=\frac{T_{n-1}}{4}, t 2=\frac{T_{n-1}}{2}, t 3=\frac{3 T_{n-1}}{4}, t 4=T_{n}$, and $t 5=\frac{5 T_{n}}{4}$. Here, $T_{n-1}$ and $T_{n}$ are the $(n-1)$ th and $n$th periods of rotation of the XCP probe as it is going down.

As can be seen from Fig. 1, $T_{I n}=T_{B n}=T_{n}, T_{Q n}=\frac{5 T_{n}}{4}-\frac{T_{n-1}}{4}$.
$\omega t_{i}=\frac{\pi}{2} i, \quad i=1, \ldots, 5$

and $T_{B n}$ are the periods of the in-phase component, quadrature component, and baseline component. Substituting Eq. (5) in Eqs. (6), (7), and (8) results in Eqs. (9), (10), and (11).

$$
\begin{aligned}
& I_{n}=-\frac{4 A}{2 \pi} T_{I n} \sin \phi+C\left(T_{n-1}-T_{n}\right)+\frac{D}{2}\left(\frac{T_{n-1}^{2}}{2}-T_{n}^{2}\right) \\
& +\frac{E}{3}\left(\frac{T_{n-1}^{3}}{4}-T_{n}^{3}\right) \\
& Q_{n}=-\frac{4 A}{2 \pi} T_{Q n} \cos \phi+\frac{5 C}{4}\left(T_{n-1}-T_{n}\right) \\
& +\frac{D}{32}\left(17 T_{n-1}^{2}-25 T_{n}^{2}\right)+\frac{E}{192}\left(53 T_{n-1}^{3}-125 T_{n}^{3}\right) \\
& B_{n}=C T_{n}+\frac{D}{2} T_{n}^{2}+\frac{E}{3} T_{n}^{3}
\end{aligned}
$$

$C, D$, and $E$ can be obtained by solving Eq. (11) for three adjacent periods, i.e. for $B_{n-1}, B_{n}$, and $B_{n+1}$. By making the circuit delay correction for Eqs. (9) and (10) based on Eq. (11), $I_{n}{ }^{\prime}$ and $Q_{n}{ }^{\prime}$, which are given by Eqs. (12) and (13), can be determined:

$$
\begin{aligned}
& I_{n}{ }^{\prime}=-\frac{4 A}{2 \pi} T_{I n} \sin \phi=I_{n}-\left[C\left(T_{n-1}-T_{n}\right)\right. \\
& \left.+\frac{D}{2}\left(\frac{T_{n-1}^{2}}{2}-T_{n}^{2}\right)+\frac{E}{3}\left(\frac{T_{n-1}^{3}}{4}-T_{n}^{3}\right)\right], \\
& Q_{n}{ }^{\prime}=-\frac{4 A}{2 \pi} T_{Q n} \cos \phi=Q_{n}-\left[\frac{5 C}{4}\left(T_{n-1}-T_{n}\right)\right. \\
& \left.+\frac{D}{32}\left(17 T_{n-1}^{2}-25 T_{n}^{2}\right)+\frac{E}{192}\left(53 T_{n-1}^{3}-125 T_{n}^{3}\right)\right] .
\end{aligned}
$$

The two components of the modulated signals, $F_{I}$ and $F_{Q}$, can be calculated based on $I_{n}{ }^{\prime}$ and $Q_{n}{ }^{\prime}$ as follows:

$$
F_{I}=-A \sin \varphi=\frac{2 \pi}{4 T_{I n}} I_{n}{ }^{\prime} F_{Q}=-A \cos \varphi=\frac{2 \pi}{4 T_{Q n}} Q_{n}{ }^{\prime} .
$$

Then, based on $F_{I}$ and $F_{Q}$, one can determine the amplitude, $A_{F}$, and phase, $\varphi_{F}$, of the modulated signals:

$$
A_{F}=\sqrt{F_{I}^{2}+F_{Q}^{2}} \varphi_{F}=\operatorname{tg}^{-1}\left(\frac{F_{I}}{F_{Q}}\right) \text {. }
$$

Next, from the gain recovery of the instrument, one can obtain the amplitudes and phases of the useful voltage and coil voltage signals:

$A_{\mathrm{E}} \angle \varphi_{\mathrm{E}}$,

$A_{\mathrm{C}} \angle \varphi_{\mathrm{C}}$.

Finally, the eastward and northward relative velocity components of the current, represented by $V_{\mathrm{Er}}$ and $V_{\mathrm{Nr}}$, respectively, can be calculated using Eqs. (16) and (17).

$V_{\mathrm{Er}}=\left(V_{\mathrm{E}}-\bar{V}_{\mathrm{E}}\right)=\frac{A_{\mathrm{E}}}{F_{z} L(1+K)} \cos \psi$

$V_{\mathrm{Nr}}=\left(V_{\mathrm{N}}-\bar{V}_{\mathrm{N}}\right)=\frac{A_{\mathrm{E}}}{F_{z} L(1+K)} \sin \psi$,

$+W \frac{F_{\mathrm{H}}}{F_{z}(1+K)}$

where $\psi=\frac{3}{2} \pi+\varphi_{\mathrm{C}}-\varphi_{\mathrm{E}}$. 


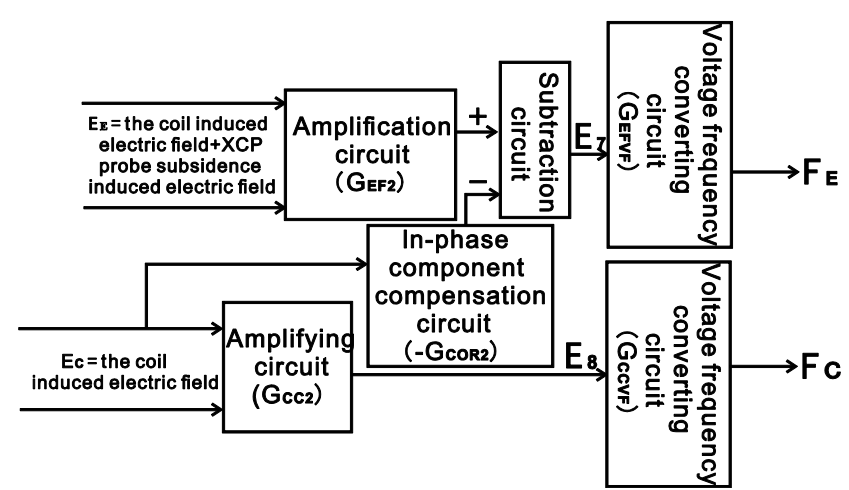

Figure 2. Circuit diagram of hardware used for data processing.

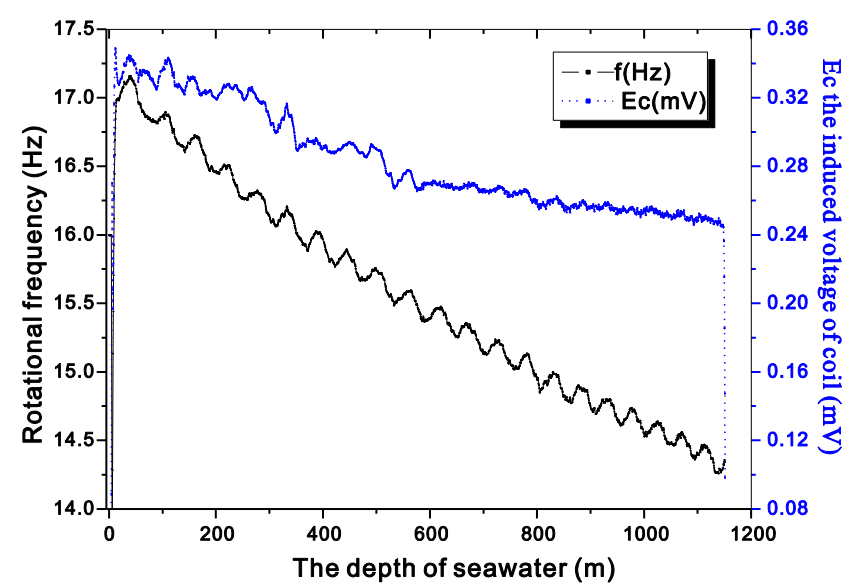

Figure 3. Rotational frequency of XCP probe and change in amplitude of coil signal, $E_{\mathrm{C}}$ (probe 1 ).

\subsection{Procedure for processing XCP ocean current data}

The procedure for processing the XCP current data is as follows:

1. Based on three adjacent periods, $B_{n-1}, B_{n}$, and $B_{n+1}$, determined from the log of the measuring instrument, solve the simultaneous equations shown below and calculate $C, D$, and $E$.

$$
\begin{aligned}
& B_{n-1}=C T_{n-1}+1 / 2 D T_{n-1}^{2}+1 / 3 E T_{n-1}^{3} \\
& B_{n}=C T_{n}+1 / 2 D T_{n}^{2}+1 / 3 E T_{n}^{3} \\
& B_{n+1}=C T_{n+1}+1 / 2 D T_{n+1}^{2}+1 / 3 E T_{n+1}^{3}
\end{aligned}
$$

2. Calculate the periods of the in-phase component, quadrature component, and baseline component, namely, $T_{I n}, T_{Q n}$, and $T_{B n}$, respectively.

$$
T_{I n}=T_{n}, T_{Q n}=5 / 4 T_{n}-1 / 4 T_{n-1}, T_{B n}=T_{n}
$$

3. Determine $F_{I}$ and $F_{Q}$ :

$$
I_{n}=-\frac{4 A}{2 \pi} T_{I n} \sin \phi+C\left(T_{n-1}-T_{n}\right)
$$

$$
\begin{aligned}
& +D\left(1 / 2 T_{n-1}^{2}-T_{n}^{2}\right) / 2+E\left(1 / 4 T_{n-1}^{3}-T_{n}^{3}\right) / 3, \\
& Q_{n}=-\frac{4 A}{2 \pi} T_{Q n} \cos \phi+C\left(T_{n-1}-T_{n}\right) 5 / 4 \\
& +D\left(17 T_{n-1}^{2}-25 T_{n}^{2}\right) 32 \\
& +E\left(53 T_{n-1}^{3}-125 T_{n}^{3}\right) / 192 .
\end{aligned}
$$

Set $I_{n}^{\prime}=-\frac{4 A T_{I n}}{2 \pi} \sin \phi$. Then, by combining Eq. (25) with Eq. (27), you get

$$
\begin{aligned}
& I_{n}^{\prime}+=-\frac{4 A T_{I n}}{2 \pi} \sin \phi=I_{n}-\left[C\left(T_{n-1}-T_{n}\right)\right. \\
& +D\left(1 / 2 T_{n-1}^{2}-T_{n}^{2}\right) / 2 \\
& \left.+E\left(1 / 4 T_{n-1}^{3}-T_{n}^{3}\right) 3\right]
\end{aligned}
$$

Set $Q_{n}^{\prime}=-\frac{4 A T_{Q n}}{2 \pi} \cos \phi$ and substitute Eq. (25) into Eq. (27):

$$
\begin{aligned}
& Q_{n}^{\prime}=-\frac{4 A T_{Q n}}{2 \pi} \cos \phi \\
& =Q_{n}-\left[C\left(T_{n-1}-T_{n}\right) 5 / 4+D\left(17 T_{n-1}^{2}-25 T_{n}^{2}\right) / 32\right. \\
& \left.+E\left(53 T_{n-1}^{3}-125 T_{n}^{3}\right) / 192\right] .
\end{aligned}
$$

Based on $I_{n}{ }^{\prime}$ and $Q_{n}{ }^{\prime}$, the two components of the modulated signals $F_{I}$ and $F_{Q}$ can be calculated as follows:

$$
\begin{aligned}
& F_{I}=-A \sin \phi=\frac{2 \pi}{4 T_{I n}} I_{n}^{\prime} \\
& =\frac{\pi}{2 T_{I n}}\left\{I_{n}-\left[C\left(T_{n-1}-T_{n}\right)+D\left(1 / 2 T_{n-1}^{2}-T_{n}^{2}\right) / 2\right.\right. \\
& \left.\left.+E\left(1 / 4 T_{n-1}^{3}-T_{n}^{3}\right) / 3\right]\right\} \\
& F_{Q}=-A \cos \phi=\frac{2 \pi}{4 T_{Q n}} Q_{n}^{\prime} \\
& =\frac{\pi}{2 T_{Q n}}\left\{Q_{n}-\left[C\left(T_{n-1}-T_{n}\right) 5 / 4\right.\right. \\
& +D\left(17 T_{n-1}^{2}-25 T_{n}^{2}\right) / 32 \\
& \left.\left.+E\left(53 T_{n-1}^{3}-125 T_{n}^{3}\right) / 192\right]\right\}
\end{aligned}
$$

4. Next, filter $F_{I}$ and $F_{Q}$ in order to get $\overline{F_{I}}$ and $\overline{F_{Q}}$ (this is done using a Bartlett, i.e. a triangle, window whose weight is $W_{n}$ ) (Zoltan, 2012; Lim and Lian, 1993):

$$
\begin{aligned}
& \overline{F_{I}}=\frac{2 \pi}{4} \sum_{n=1}^{\mathrm{Nav}} W_{n} I_{n}^{\prime} / T_{I n}=\sum_{n=1}^{\mathrm{Nav}} W_{n} F_{\mathrm{In}}, \\
& \overline{F_{Q}}=\frac{2 \pi}{4} \sum_{n=1}^{\mathrm{Nav}} W_{n} Q_{n}^{\prime} / T_{Q n}=\sum_{n=1}^{\mathrm{Nav}} W_{n} F_{Q n} .
\end{aligned}
$$




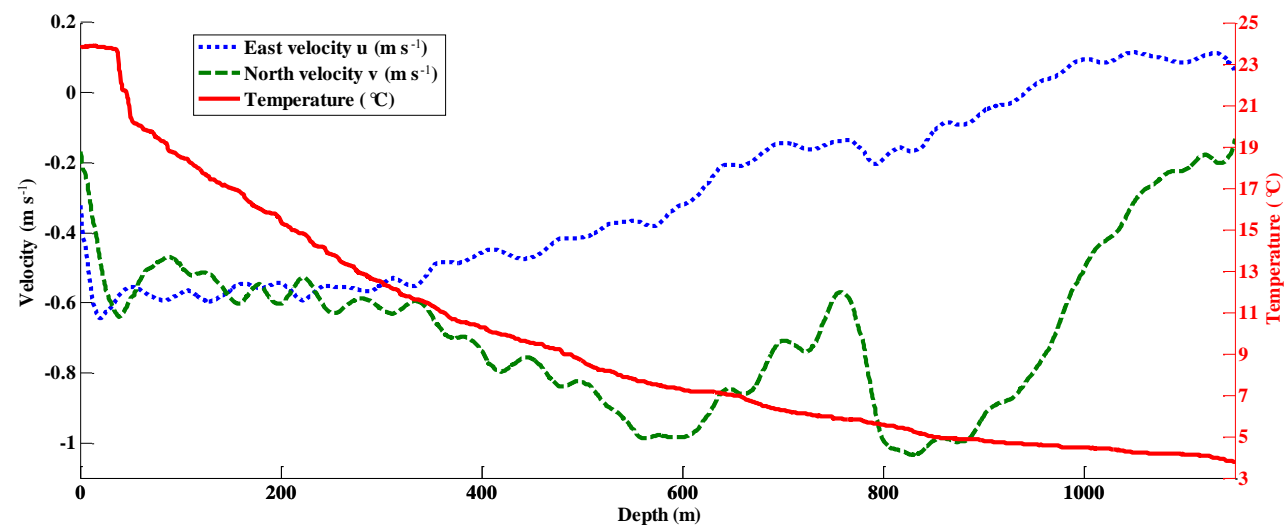

Figure 4. Ocean current velocity and temperature data as obtained using XCP probe (probe 1).

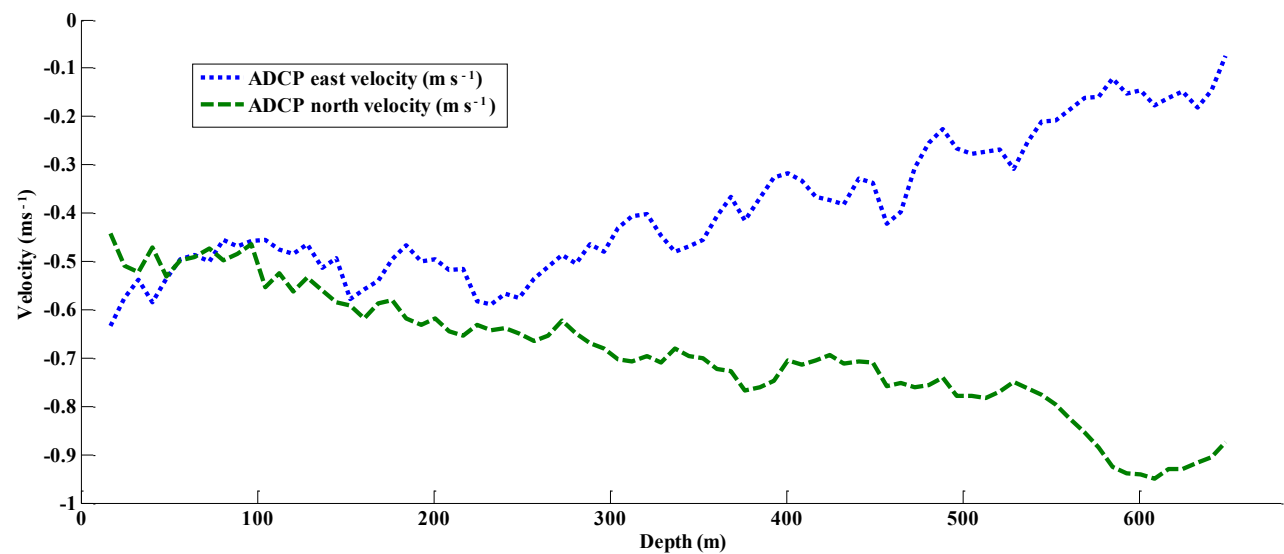

Figure 5. Ocean current velocity data $\left(21^{\circ} 59.42^{\prime} \mathrm{N}, 118^{\circ} 10.31^{\prime} \mathrm{E}\right)$ as obtained using ADCP.

5. Based on the two components calculated above, the amplitude, $A_{F}$, and phase, $\varphi_{F}$, of the modulated signals can be obtained:

$$
\begin{aligned}
& A_{F}=\left({\overline{F_{I}}}^{2}+{\overline{F_{Q}}}^{2}\right)^{1 / 2}, \\
& \varphi_{F}=\tan ^{-1} \frac{-\overline{F_{I}}}{-\overline{F_{Q}}} F=A_{F} \angle \varphi_{F} .
\end{aligned}
$$

6. The amplitude and phase of the useful voltage signal and coil voltage signal are calculated using the procedure described in steps 3-5. It can be concluded that the amplitude and phase of the electric-field-related current signal and coil signal are $F_{\mathrm{E}}=A_{\mathrm{E}} \angle \varphi_{\mathrm{E}}$ and $F_{\mathrm{C}}=$ $A_{\mathrm{C}} \angle \varphi_{\mathrm{C}}$, respectively.

7. The circuit diagram of the hardware used for data processing is shown in Fig. 2. One can determine the original electric field and coil electric field as follows:

$$
\begin{aligned}
E_{\mathrm{E}} & =\frac{F_{\mathrm{E}}}{G_{\mathrm{EFVF}} G_{\mathrm{EF} 2}}-E_{\mathrm{C}} \frac{G_{\mathrm{COR} 2}}{G_{\mathrm{EF} 2}}, \\
E_{\mathrm{C}} & =\frac{F_{\mathrm{C}}}{G_{\mathrm{CCVF}} G_{\mathrm{CC} 2}},
\end{aligned}
$$

where $\quad G_{\mathrm{EF} 2}=\frac{E_{7}}{E_{\mathrm{E}}}, \quad G_{\mathrm{COR} 2}=\frac{E_{7}}{E_{\mathrm{C}}}, \quad G_{\mathrm{CC} 2}=\frac{E_{8}}{E_{\mathrm{C}}}$, $G_{\mathrm{EFVF}}=\frac{F_{\mathrm{E}}}{E_{7}}(\mathrm{~Hz} / V)$, and $G_{\mathrm{CCVF}}=\frac{F_{\mathrm{C}}}{E_{8}}(\mathrm{~Hz} / V)$.

8. Calculate the northward speed and the eastward speed:

$$
\begin{aligned}
& u=\frac{A_{\mathrm{E}}}{F_{Z} L(1+K)} \cos \psi, \\
& v=-\frac{A_{\mathrm{E}}}{F_{Z} L(1+K)} \sin \psi+W \frac{F_{\mathrm{H}}}{F_{Z}(1+K)},
\end{aligned}
$$

where $\psi=\frac{3}{2} \pi+\varphi_{\mathrm{C}}-\varphi_{\mathrm{E}}$.

Based on the velocity model, which uses $W=-2 \times a \times$ $t-b$, the relation between the depth of the XCP probe in the ocean and time is $Z=-1 \times\left(c+b \times t+a \times t^{2}\right)$, where $a=$ $-3.5 \times 10^{-4}, b=3.85$, and $c=3.1$. This is the experience value of the development of the XCP probe.

\section{Results and discussion}

Because laboratory simulations of the marine environment are difficult to perform, we performed a sea test using the 
$\mathrm{XCP}$ in order to verify the accuracy of the proposed method for processing the XCP current data. An XCP probe (probe 1) was combined with an XCP buoy and released in the test area. The location of the release point was $21^{\circ} 59.420^{\prime} \mathrm{N}$, $118^{\circ} 10.310^{\prime} \mathrm{E}$, and data were collected up to a maximum depth of $1.151 \mathrm{~m}$. The changes in the rotational frequency and coil signal $(\mathrm{Ec})$ of the probe during sinking are shown in Fig. 3. The collected Ec data were processed to obtain the ocean current velocity data, which were then combined with the collected temperature data to plot the curves shown in Fig. 4.

The data shown in Figs. 4 and 5 were collected at a location $44.3^{\circ}$ east by south of Shantou, Guangdong Province, China, and approximately $215 \mathrm{~km}$ away from Shantou, Guangdong Province, China. For comparison, Fig. 5 shows the ocean current velocity data as measured by an acoustic Doppler current profiler (ADCP) (Zoltan, 2012). The ADCP used was an OS-75K ADCP supplied by RDI and had a maximum profiling depth of $700 \mathrm{~m}$ (Liu, 2016). A comparison of Figs. 4 and 5 shows that the ocean current velocity as measured by the XCP was generally consistent with that measured by the ADCP. From $16 \mathrm{~m}$ BSL to $600 \mathrm{~m}$ BSL, the velocity of the eastward ocean current changed gradually from -0.6 to $-0.1 \mathrm{~m} \mathrm{~s}^{-1}$, while the velocity of the northward ocean current changed gradually from -0.5 to $-0.96 \mathrm{~m}, \mathrm{~s}^{-1}$. The current at the top layer ran southwestward in a direction approximately parallel to the coastal line in Guangdong Province, China.

\section{Conclusions}

Based on the theoretical principles underlying the processing of current data obtained using an XCP, in this study, we developed a method for processing XCP data in order to improve the accuracy of the measurements. In addition, a sea test was performed to evaluate the accuracy of the proposed method. Relationships derived based on theoretical studies were used to process the data collected from the sea test and to plot the current velocity data. Moreover, the current velocity data obtained using the XCP were compared with those obtained using an ADCP. It was found that the trends in these two sets of velocity data were essentially consistent, confirming the accuracy of the proposed method for processing XCP current data.

Data availability. No data sets were used in this article.

Competing interests. The authors declare that they have no conflict of interest.
Acknowledgements. This work was supported by the Fundamental Research Funds for the Central Universities of China (no. 2652014070), the National Natural Science Foundation of China (no. 41574131), and the National 863 Program of China (no. 2012AA061102).

Edited by: L. Vazquez

Reviewed by: two anonymous referees

\section{References}

Chave, A. D. and Luther, D. S.: Low frequency motionally-induced electromagnetic fields in the ocean, J. Geophys. Res., 95, 7185$7200,1990$.

Chen, W. Y., Zhang, R., Liu, N., Zhang, M. M., and Tao, J. L.: Numerical simulation the flow field of expendable current profiler probe, Sci. Technol. Rev., 28, 62-65, 2010.

Chen, W. Y., Zhang, R., Liu, N., and Zhang, M. M.: Numerical study on the influence of rotating to the movement characteristics of XCP probe, Ocean Technol., 30, 61-63, 2011.

Crews, A. and Futterman, J.: Geomagnetic micropulsations due to the motion of ocean waves, J. Geophys. Res., 67, 299-306, 1962.

Gandolfi, A., Nobili, C., Prudenziati, M., and Taroni, A.: Voltageto-frequency conversion of signals supplied by physical-quantity sensors, IEEE T. Ind. El. Con. In., 4, 107-114, 1972.

Hisayoshi, S. and Hisashi, U.: Motional magnetotellurics by long oceanic waves, Geophys. J. Int., 201, 390-405, 2015.

Lim, Y. C. and Lian, Y.: The optimum design of one- and twodimensional FIR filters using the frequency-response masking technique, IEEE Trans. Circuits Syst., 40, 88-95, 1993.

Lin, C. S. and Ren, D. K.: Calculation of electromagnetic field induced by ocean current, Journal of Naval University of Engineering, 15, 19-22, 2003.

Liu, N. and He, H. K.: Study on the theory of expendable current profiler measurement, Ocean Technol., 29, 8-11, 2010.

Liu, N., Li, Y. J., and Zhu, G. W.: A kind of fast expendable current profiler measure production, Ocean Technol., 26, 27-31, 2007.

Liu, Y. X.: Review on development of ADCP technology and its application, Hydrographic Surveying and Charting, 36, 45-19, 2016.

Larsen, J. C. and Sanford, T. B.: Florida current volume transports from voltage measurements, Science, 227, 302-304, 1985.

Wang, Y. B., Chen, W. D., and Du, Y.: Effect of ocean wave on magnitude of underwater electromagnetic field, J. Trop. Oceanogr., 24, 37-40, 2005.

Wu, J. S. and Chen, Y.: Study on the waves by the electromagnetic field induced by the waves in the geomagnetic field, Mar. Sci. 6, 26-30, 1991.

Yan, X. W., Yan, H., and Xiao, C. H.: Research on model of induce magnetic vector of ocean waves, Hydrographic Surveying and Charting, 31, 8-11, 2011.

Zhang, Q. S., Deng, M., and Wang, Q.: Dynamic data transmission technique for expendable current profiler, Adv. Mat. Res., 220, 436-440, 2011.

Zhang, Q. S., Xiao, Z., Xinyue, Z. et al.: Influence of Expendable Current Profiler Probe on Induced Electric Field of Ocean Currents, Math. Probl. Eng., 9812929, doi:10.1155/2016/9812929, 2016 . 
Zhang, Z. L., Wei, W. B., Liu, B. H., Deng, M., and Jin, S.: Theoretical calculation of electromagnetic field generated by ocean waves, Acta Oceanol. Sin., 30, 42-46, 2008.
Zoltan, B. S.: Using motionally-induced electric signals to indirectly measure ocean velocity: Instrumental and theoretical developments, Prog. Oceanogr., 96, 108-127, 2012. 